\title{
Les enjeux de la mémoire dans la littérature et les arts contemporains de la République démocratique du Congo, dir. É. BREZAULT
}

\section{Elena Pessini}

\section{(2) OpenEdition}

\section{Journals}

\section{Édition électronique}

URL : https://journals.openedition.org/studifrancesi/32348

DOI : $10.4000 /$ studifrancesi.32348

ISSN : 2421-5856

Éditeur

Rosenberg \& Sellier

\section{Édition imprimée}

Date de publication : 1 août 2020

Pagination : 446-448

ISSN : 0039-2944

\section{Référence électronique}

Elena Pessini, « Les enjeux de la mémoire dans la littérature et les arts contemporains de la République démocratique du Congo, dir. É. BREZAuLt », Studi Francesi [En ligne], 191 (LXIV | II) | 2020, mis en ligne le 01 septembre 2020, consulté le 18 septembre 2021. URL : http://journals.openedition.org/ studifrancesi/32348; DOI : https://doi.org/10.4000/studifrancesi.32348

Ce document a été généré automatiquement le 18 septembre 2021.

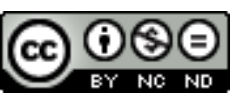

Studi Francesi è distribuita con Licenza Creative Commons Attribuzione - Non commerciale - Non opere derivate 4.0 Internazionale. 


\title{
Les enjeux de la mémoire dans la littérature et les arts contemporains de la République démocratique du Congo, dir. É. BREZAULT
}

\author{
Elena Pessini
}

\section{RÉFÉRENCE}

Les enjeux de la mémoire dans la littérature et les arts contemporains de la République démocratique du Congo, dir. É. BREZAULT, "Francofonia. Studi e ricerche sulle letterature di lingua francese" 76, Primavera 2019, 222 pp.

1 Le titre du numéro daté printemps 2019 de la revue "Francofonia" que nous présentons ici indique clairement que les interventions qui y sont réunies ne convoquent pas seulement la littérature de la République Démocratique du Congo mais également d'autres formes d'art contemporain, en l'occurrence le cinéma et la bande dessinée. Éloïse BREZAult, qui a dirigé ce recueil, trace dans son introduction la genèse de l'ouvrage qui a eu comme point de départ un panel organisé par l'African Literature Association à Yale University en 2017 autour de «La question mémorielle dans les littératures actuelles de la République Démocratique du Congo. Tout était parti d'un constat: comment comprendre la prédominance du temps présent dans la littérature actuelle du Congo et l'absence de traces du passé» (p. 9). Une première partie, la plus riche en contributions, est entièrement consacrée à la littérature et tous les travaux qui y sont présentés ont le mérite à la fois de problématiser le rapport que les textes entretiennent avec l'Histoire, au passé, à la mémoire et en même temps d'offrir une analyse très fouillée des récits, des romans qui représentent le mieux le visage de la littérature de la République démocratique du Congo de l'extrême contemporain. En particulier, les chercheurs s'interrogent sur le «présentisme», pour employer le terme 
que définit Hartog dans son ouvrage Régimes d'historicité, ouvrage souvent cité comme référence par les auteurs du dossier, cette obsession du présent que l'on trouve dans les romans, les films et également les bandes dessinées congolais. Ce poids du présent appelle évidemment toute une série de considérations sur la véritable nature de ce déploiement de l'hic et nunc et surtout sur son rapport avec le passé, passé de colonisation, de violence, de dictature pour la République démocratique du Congo mais aussi sur le rôle que recouvre la mémoire dans les différents genres de narration fictionnelle. Une mémoire inévitablement «diasporique et fragmentaire» (p. 11) mise en place, il ne s'agit pas d'un détail, par des écrivains qui, pour la plupart, écrivent hors des frontières de leur pays d'origine. La grande question à laquelle veulent répondre les chercheurs qui alternent leurs voix dans le dossier de la revue est la suivante, exprimée en introduction par Éloïse Brezault: «comment les écrivains congolais disent-ils leur pays. [...] De quelle manière les écrivains et les artistes congolais entretiennent-ils le travail de mémoire, pour reprendre les propos de Ricœur, plutôt que de lui donner une forme figée comme le remarquait Michael Rothberg?» (p. 11).

2 L'article de Kasongo Mulenda KAPANGA, intitulé Derrière l'immédiateté, les points nodaux dans le récit congolais: deux générations d'écrivains, figure très justement en ouverture du premier volet littéraire de ce travail de recherche puisqu'il met en place une série de jalons chronologiques tant du point de vue de l'histoire littéraire du pays que de l'Histoire tout court. À travers une perspective diachronique, il s'agit de faire dialoguer deux générations d'écrivains, la génération du Mont Amba, qui comprend en particulier des écrivains tels que Mudimbe, Ngal, Ngandu-Nkashama et une génération plus récente, avec, entre autres Fiston Mwanza et In Koli Jean Bofane. L'analyse détaillée de deux romans $L a$ Re-production de Thomas Mpoyi-Buatu publié en 1986 et Tram 83, publié en 2014 de Fiston Mwanza Mujila va permettre de creuser la problématique et de montrer que si le passé occupe dans les deux cas la matière du roman, d'une génération à l'autre le dialogue avec le présent se met en place avec des instances qui sont sensiblement différentes. La notion de nostalgie est au cœur de l'article de Karen BOUWER intitulé Nostalgie et mémoire en transit: «Samantha à Kinshasa» de Marie-Louise Mumbu, une nostalgie réparatrice, «restorative nostalgia» et une nostalgie réfléchie, «reflective nostalgia», théorisées par Svetlana Boym dans son ouvrage The Future of Nostalgia (2001). L'héroïne du roman, depuis l'avion dans lequel elle quitte son pays d'origine évoque sa ville, Kinshasa, et ses habitants. Dans ce non-lieu du moyen de transport aérien, passé et présent s'entrechoquent et le roman donne vie à un kaléidoscope spatial et temporel où l'exercice de la mémoire joue un rôle essentiel. Le roman Tram 83, déjà évoqué dans la première contribution revient dans l'article de Katie TIDMASH qui lui est entièrement consacré: “Tram 83", trains, traumatismes et nationalisme dans les écrits de Fiston Mwanza Mujila. Texte touffu et riche, ouvrage incontournable de la littérature congolaise contemporaine, il mérite l'attention des spécialistes qui se sont fixé l'objectif de réfléchir sur les thématiques qui ont trait à la représentation de la mémoire car il aborde une question essentielle: «comment écrire son pays et son histoire quand se posent le besoin d'assimiler le passé difficile d'un pays comme le Congo, les attentes capricieuses d'un lectorat étranger, et le risque de confirmer les clichés de l'Afrique de la misère ou de la violence?» (p. 52). L'analyse de Tidmash montre bien que Tram 83 interroge les rapports entre le passé colonial et la modernité en proie à la mondialisation mais propose surtout une forme romanesque, une forme littéraire, particulièrement originale, capable d'échapper aux balises de la littérature dite «du dehors» et de trouver un point d'équilibre entre évocation du passé 
et face à face avec le présent. Éloïse BREZAULT, avec Mondialisation et 'Afrocontemporanéité' dans "Congo Inc." ou comment repenser la mémoire coloniale et la modernité, concentre, elle aussi sa recherche sur un ouvrage en particulier, le roman de l'écrivain In Koli Jean Bofane, publié chez Actes Sud en 2014 dont le titre complet est Congo Inc. Le testament de Bismarck. L'analyse qu'elle propose de ce récit conclut qu'à travers le personnage principal de l'histoire, Isookanga, le romancier problématise un dépassement de la dichotomie entre passé et présent, oubli du passé et obsession du présent, dans une «fusion entre savoirs traditionnels et modernes, sans tomber dans l'aliénation du passé ou la peur du futur» (p. 80). Un autre roman très récent a focalisé l'intérêt d'Elara BERTHO qui, dans son article intitulé Cacophonies de la mémoire et éblouissements impériaux soumet l'ouvrage Généalogie d'une banalité de Sinzo Aanza à son analyse qui nous entraine dans une "ville capharnaüm», lieu de toutes les illusions, lieu emblématique de la mondialisation et de tous ses excès, symbole et archétype, à travers lequel l'écrivain peut élever la voix de sa mise en cause de la réalité contemporaine. Deux romans de Blaise Ndala, J'irai danser sur la tombe de Senghor (2014) et Sans capote ni kalachnikov (2017) font l'objet du travail de Jean DE DIEU ITSIEKI PUTU BASEY qui a pour titre La rumba des mots ou la 'prose combat' de Blaise Ndala: dire le naufrage de l'histoire et décrypter les origines d'une apocalypse joyeuse et qui ferme la section consacrée à la littérature. Les deux romans annoncent par leur titre la couleur de leur contenu qui ménage une grande place à l'humour, un humour qui n'empêche pas une réflexion, ou qui même la favorise, sur le rapport entre la réalité de l'extrême contemporain et des moments historiques fondateurs ou fondamentaux sur lesquels il est essentiel de revenir.

3 Comme nous avons annoncé en ouverture, un regard sur le cinéma est présent même s'il ne s'agit que d'un article, Congolese cinema today: memories of the present in Kinshasa new wave cinema (2008-2018), signé par Matthias DE GROOF et Alessandro JEDLOWSKI. Parfaitement cohérente avec l'ensemble des problématiques abordées dans le numéro de la revue, cette contribution a le mérite de souligner les spécificités de la production cinématographique congolaise contemporaine, une spécificité qui s'affiche dans les procédés de gommage systématique des références au passé précolonial, colonial et postcolonial.

4 Une série d'interviews conclut l'ouvrage mais elles ne sont pas à considérer comme une simple annexe. Les deux entretiens qui concernent des écrivains, respectivement Fiston Nasser Mwanza Mujila (Le théâtre-conte chez Fiston Nasser Mwanza Mujila: une histoire du présent) et In Koli Jean Bofane ("Congo Inc." ou les aléas de la mondialisation), réalisés par Éloïse BREZAULT, apportent un éclairage significatif sur les œuvres des deux romanciers et permettent au lecteur de mettre en place un dialogue fécond avec les articles qui précèdent. L'entretien réalisé par Sandra FEDERICI ouvre sur le monde de la bande dessinée congolaise, un monde très riche en créateurs et en nouveautés. Il s'agit en réalité de deux interviews au dessinateur Barly Baruti, regroupées sous le titre: $L a$ mémoire historique comme choix pour l'affirmation dans le champ de la bande dessinée, qui se focalisent en particulier sur l'album Madame Livingstone. Congo, la Grande Guerre (2014), qui annonce bien l'importance du rapport au passé.

5 Ce numéro de "Francofonia" est à conseiller à la fois aux spécialistes pour les approches savantes qu'ils pourront y trouver mais également pour un lectorat qui souhaiterait découvrir les multiples facettes d'une culture fascinante et dynamique. 